\title{
COHOMOLOGY OF FINITE COVERS
}

\author{
BY
}

\section{ALLAN CALDER}

ABSTRACT. For a finite dimensional CW-complex, $X$, and $q>1$, it is shown that the qth Čech cohomology group based on finite open covers of $X, H_{f}^{q}(X)$, is naturally isomorphic to $H^{q}(X)$, the qth Čech cohomology of $X$ (i.e. based on locally finite covers), and for reasonable $X, H^{1}(X)$ can be obtained algebraically from $H_{f}^{1}(X)$.

The Čech cohomology functor $H_{f}^{*}$ based on finite open covers [2], [5] is not a cohomology theory. Dowker showed that $H_{f}^{1}$ of the real line is nontrivial and so $H_{f}^{1}$ does not satisfy the homotopy axiom [3]

In this paper I show that for finite dimensional CW-complexes, $H_{f}^{q}$ is naturally isomorphic to the usual Čech cohomology functor $H^{q}$, i.e. based on locally finite covers, when $q>1$ and for a nice space $X, H^{1}(X)$ can be obtained algebraically from $H_{f}^{1}(X)$. This greatly extends previous results [1].

I wish to thank E. H. Brown for very useful conversations concerning aspects of this work.

Let $\beta$ be the Stone-Čech functor from the category of normal spaces and maps (continuous functions) to the category of compact ( $\Rightarrow$ Hausdorff) spaces and maps. In other words, $\beta X$ is the Stone-Cech compactification of $X$ and $\beta(f: X \rightarrow Y): \beta X \rightarrow \beta Y$ is the unique "extension" of $f$. We shall also use $\beta$ to denote the embedding of $X$ into $\beta X$.

For a space $X$ and a topological group $Y,[X, Y]$ denotes the set of homotopy classes of maps from $X$ to $Y$ together with the group structure induced by $Y$.

A precise definition of Čech cohomology based on finite open covers can be found in [5, Chapter 9]. It is sufficient here to know that for a normal space $X$, the embedding $\beta: X \rightarrow \beta X$ induces a natural isomorphism from $H_{f}^{*}(X)$ to $H^{*}(\beta X),[5$, p. 282]

For paracompact spaces, $H^{q}(-; \pi)$ is naturally isomorphic to $[-, K(\pi, q)]$, where $\pi$ is the coefficient group and $K(\pi, q)$ is an Eilenberg-Mac Lane space of type $(\pi, q),[7, \mathrm{p} .423]$. So for normal spaces, $H_{f}^{q}(X ; \pi)$ is naturally isomorphic to $[\beta X, K(\pi, q)]$.

LEMMA 1. For $X$ a finite dimensional $C W$-complex, $\pi$ a finitely generated

Received by the editors March 28, 1975.

AMS (MOS) subject classifications (1970). Primary 55 B05. 
abelian group and $q>0$, the embedding $\beta: X \rightarrow \beta X$ induces an epimorphism $\beta^{*}$ : $[\beta X, K(\pi, q)] \rightarrow[X, K(\pi, q)]$.

Proof. Let $\langle\cdot\rangle$ denote "equivalence class of", then $\beta^{*}\langle f\rangle=\langle f \beta\rangle$.

Since $\pi$ is finitely generated and $q>0$, we can choose $K(\pi, q)$ to be a CW-complex of finite type, i.e. with compact skeleta. Let $\langle f\rangle \in[X, K(\pi, q)]$. By the cellular approximation theorem we can assume that $f$ is cellular. Since $X$ is finite dimensional $f(X)$ is contained in a compact subspace of $K(\pi, q)$ and so $f$ can be extended to a map $F: \beta X \rightarrow K(\pi, q)$. Now $\beta^{*}\langle F\rangle=\langle f\rangle$.

To prove the main theorem we must show that for finite dimensional $\mathrm{CW}$ complexes and $q>1$, the kernel of $\beta^{*}$ is trivial. The key to establishing that is the following

LEMMA 2. Let $p: E \rightarrow B$ be a principal $G$-bundle with $G$ a $C W$-complex of finite type and $B$ compact. If $X$ is a finite dimensional $C W$-complex and $f$ : $X \rightarrow E$ is a map then there is a map $g: X \rightarrow E$ such that $p f=p g$ and $\overline{g(X)}$ is compact.

Proof. Let $\left\{V_{i}\right\}$ be a finite atlas for the bundle, with coordinate functions $\varphi_{i}: G \times V_{i} \rightarrow p^{-1} V_{i}$. Let $\left\{U_{i}\right\}$ be a shrinking of $\left\{V_{i}\right\}$, i.e. $\left\{U_{i}\right\}$ covers $B$ and $\bar{U}_{i} \subset V_{i}$.

Let $t_{i j}: V_{i} \cap V_{j} \rightarrow G$ be the coordinate transformations and let $G^{k}$ denote the $k$-skeleton of $G$. Since $t_{i j}\left(\bar{U}_{i} \cap \bar{U}_{j}\right)$ is compact it is contained in $G^{m(i, j)}$ for some $m(i, j)$. Put $m=\max _{i, j}\{m(i, j)\}$ and for each $k$, choose $m(k)$ such that

$$
G^{m} G^{k}=\left\{g g^{\prime}: g \in G^{m} \text { and } g^{\prime} \in G^{k}\right\} \subset G^{m(k)}
$$

and $m(k)>k$.

Let $E^{k}=\bigcup_{i} \varphi_{i}\left(G^{k} \times \bar{U}_{i}\right)$. Then $E^{k}$ is compact and

$$
E^{k} \cap p^{-1} U_{i} \subset \varphi_{i}\left(G^{m(k)} \times U_{i}\right) .
$$

[For if $y \in E^{k} \cap p^{-1} U_{i}$ then $y \in \varphi_{j}\left(G^{k} \times \bar{U}_{j}\right)$ for some $j$ and $p y \in U_{i} \cap \bar{U}_{j}$. So if $\varphi_{j}^{-1} y=(g, p y) \in G^{k} \times \bar{U}_{j}$ then $\varphi_{i}^{-1} y=\left(t_{i j}(p y) g, p y\right) \in G^{m} G^{k} \times U_{i} \subset$ $G^{m(k)} \times U_{i}$.]

Subdivide $X$ so that for each cell $u$ in $X$ there is an $i$ such that $p f \bar{u} \subset U_{i}$. Let $X^{n}$ denote the $n$-skeleton of $X$ in this subdivision and let $M^{n}=(X \times\{0\})$ $\cup\left(X^{n} \times I\right)$, where $I=[0,1]$. Let $q_{i}=\theta \varphi_{i}^{-1}: p^{-1} V_{i} \rightarrow G$, where $\theta: G \times V_{i}$ $\rightarrow G$ is the projection. For $v \in X^{0}$, let $\lambda_{v}: I \rightarrow G$ be a path from $q_{i} f v$ to a 0 -cell of $G$, where $p f v \in U_{i}$.

Define a map $F^{0}: M^{0} \rightarrow E$ by

$$
\begin{aligned}
& F^{0}(x, 0)=f x, \quad x \in X, \\
& F^{0}(v, t)=\varphi_{i_{v}}\left(\lambda_{v} t, p f v\right), \quad v \in X^{0}, \quad t \in I \text { and } p f v \subset U_{i_{v}} .
\end{aligned}
$$


Then $p F^{0}(x, t)=p f x$ and $F^{0}\left(X^{0} \times\{1\}\right) \subset E^{0}$.

Assume that there exists a map $F^{n-1}: M^{n-1} \rightarrow E$ such that $p F^{n-1}(x, t)=$ $p f x$ and $F^{n-1}\left(X^{n-1} \times\{1\}\right) \subset E^{k}$ for some $k \geqslant n$. For each $n$-cell $u$ in $X$, choose $i_{u}$ such that $p f \bar{u} \subset U_{i_{u}}$. Then

$$
F^{n-1}(u x\{0\}) \subset p^{-1} U_{i_{u}}
$$

and

$$
F^{n-1}(\dot{u} x\{1\}) \subset E^{k} \cap p^{-1} U_{i_{u}} \subset \varphi_{i_{u}}\left(G^{m(k)} \times U_{i_{u}}\right) .
$$

[ $\dot{u}$ denotes the boundary of $u$ in $X$.] Let

$$
h_{u}=q_{i_{u}} F^{n-1} \mid(u x\{0\}) \cup(\dot{u} \times I) .
$$

Then $h_{u}(\dot{u} \times\{1\}) \subset G^{m(k)}$. Since $m(k) \geqslant n, \pi_{n}\left(G, G^{m(k)}\right)=0$ and therefore $h_{u}$ can be extended to a map $H_{u}: u \times I \rightarrow G$ such that $H_{u}(u \times\{1\}) \subset G^{m(k)}$. Define $F^{n}: M^{n} \rightarrow E$ by $F^{n} \mid M^{n-1}=F^{n-1}$ and $F^{n}(x, t)=\varphi_{i_{u}}\left(H_{u}(x, t), p f x\right)$, for $x \in u$. Then $F^{n}$ is a map such that $p F^{n}(x, t)=p f x, F^{n} \mid M^{n-1}=F^{n-1}$ and $F^{n}\left(X^{n} \times\{1\}\right)$ is contained in $E^{m(k)}$. So by induction such a map exists for all $n$.

Define $g: X \rightarrow E$ by $g x=F^{n}(x, 1)$ for all $x \in X^{n}$. Then $p f=p g$ and $\overline{g\left(X^{n}\right)}$ is compact for each $n$. Thus if $X$ is finite dimensional $\overline{g(X)}$ is compact.

THEOREM 1. For $X$ a finite dimensional $C W$-complex, $\pi$ a finitely generated abelian group and $q>1$,

is an isomorphism.

$$
\beta^{*}: H_{f}^{q}(X ; \pi) \rightarrow H^{q}(X ; \pi)
$$

Proof. Assume $K(\pi, q)$ is of finite type and let $p: E \rightarrow K(\pi, q)$ be the universal bundle. Let $\langle f\rangle \in \operatorname{ker} \beta^{*}$. Then $f \beta$ can be lifted to a map $f^{\prime}: X \rightarrow E$. Now $f \beta(X) \subset f(\beta X)$ which is compact and so contained in the $k$-skeleton $K^{k}$ of $K(\pi, q)$, for some $k$. Let $E^{\prime} \rightarrow K^{k}$ be the $K(\pi, q-1)$-bundle over $K^{k}$ obtained by restricting $p: E \rightarrow K(\pi, q)$. We can now apply Lemma 2 to $f^{\prime}$ restricted to $E^{\prime}$ to obtain a map $g: X \rightarrow E$ such that $\overline{g(X)}$ is compact and $p g=f \beta$. Now $g$ can be extended to a map $g^{\prime}: \beta X \rightarrow E$ and $p g^{\prime}=f$. So $f$ is null homotopic and hence $\operatorname{ker} \beta^{*}=0$. The result now follows by Lemma 1 .

We now consider the case $q=1$, and $X$ a normal space.

Let $j: X \rightarrow C X$ be the embedding of $X$ as the base of the cone $C X$ on $X$. Then $\alpha=\beta j: \beta X \rightarrow \beta C X$ is also an embedding. Consider the sequence

$$
\left[\beta C X, S^{1}\right] \stackrel{\alpha^{*}}{\rightarrow}\left[\beta X, S^{1}\right] \stackrel{\beta^{*}}{\rightarrow}\left[X, S^{1}\right],
$$

where $\alpha^{*}$ is induced by $\alpha$ and $S^{1}$ is the circle. If $\langle f\rangle \in \operatorname{im} \beta^{*} \alpha^{*}$, then $f$ can be extended to $\beta C X$ and thus to $C X$ and so is null homotopic. So $\operatorname{im} \alpha^{*} \subset \operatorname{ker} \beta^{*}$. 
If $\langle g\rangle \in \operatorname{ker} \beta^{*}$, then $g \beta$ is null homotopic, so can be extended to $C X$ and hence to $\beta C X$. Thus $\operatorname{ker} \beta^{*}=\operatorname{im} \alpha^{*}$.

Because $C X$ is contractible it follows from [4], or see [8, p. 225], that $\left[\beta C X, S^{1}\right]$ is isomorphic to the quotient of the additive group of real valued maps on $C X$ by the subgroup of bounded maps. That is a divisible group [6, p. 163] and so $\operatorname{im} \alpha^{*}$ is a divisible group. Hence the short exact sequence

$$
0 \rightarrow \operatorname{ker} \beta^{*} \rightarrow\left[\beta X, S^{1}\right] \stackrel{\beta^{*}}{\rightarrow}\left[X, S^{1}\right] \rightarrow 0
$$

splits. ( $\beta^{*}$ is onto since $S^{1}$ is compact.)

Thus if $H^{1}(X) \cong\left[X, S^{1}\right]$ is reduced, i.e. has no divisible subgroups, $\operatorname{ker} \beta^{*}$ is the unique maximal divisible subgroup, $[6, \mathrm{p} .164]$, of $H_{f}^{1}(X) \cong\left[\beta X, S^{1}\right]$. In particular this will be the case if $X$ has the homotopy type of a CW-complex with a finite 1-skeleton. Hence we have proved the following result.

THEOREM 2. If $X$ is a normal space and $H^{1}(X)$ is a reduced group then $H^{1}(X) \cong H_{f}^{1}(X) / G$, where $G$ is the maximal divisible subgroup of $H_{f}^{1}(X)$.

Theorems 1 and 2 can easily be extended to a relative $C W$-complex $(X, A)$ since $\beta(X / A)$ is homeomorphic to $\beta X / \beta A$.

\section{REFERENCES}

1. A. Calder, On the cohomology of $\beta R^{n}$, Quart. J. Math. Oxford Ser. (2) 25 (1974), $385-394$

2. E. Čech, Théorie générale de l'homologie dans un espace quelconque, Fund. Math. 19 (1932), 149-183.

3. C. H. Dowker, Mapping theorems for non-compact spaces, Amer. J. Math. 69 (1947), 200-242. MR 8, 594.

4. S. Eilenberg, Transformations continues en circonférence et la topologie du plan, Fund. Math. 26 (1936), 61-112.

5. S. Eilenberg and N. Steenrod, Foundations of algebraic topology, Princeton Univ. Press, Princeton, N. J., 1952. MR 14, 398.

6. A. G. Kuroš, The theory of groups, 2nd ed., GITTL, Moscow, 1953; English transl., Chelsea, New York, 1955. MR 15, 501; 17, 124.

7. C. N. Lee and F. Raymond, Čech extensions of contravariant functors, Trans. Amer. Math. Soc. 133 (1968), 415-434. MR 38 \#2767.

8. G. T. Whyburn, Analytic topology, Amer. Math. Soc. Colloq. Publ., vol. 28, Amer. Math. Soc., Providence, R. I., 1942. MR 4, 86.

DEPARTMENT OF MATHEMATICS, BIRKBECK COLLEGE, UNIVERSITY OF LONDON, LONDON, ENGLAND

DEPARTMENT OF MATHEMATICS, UNIVERSITY OF MISSOURI, ST. LOUIS, MISSOURI 63121 